Acta Crystallographica Section D

\title{
Biological
}

Crystallography

ISSN 0907-4449

Editors: E. N. Baker and Z. Dauter

\section{The crystal structure analysis of the relative binding of cisplatin and carboplatin in a mixture with histidine in a protein studied at 100 and $300 \mathrm{~K}$ with repeated X-ray irradiation}

\author{
John R. Helliwell and Simon William Maurice Tanley
}

Acta Cryst. (2013). D69, 121-125

Copyright (C) International Union of Crystallography

Author(s) of this paper may load this reprint on their own web site or institutional repository provided that this cover page is retained. Republication of this article or its storage in electronic databases other than as specified above is not permitted without prior permission in writing from the IUCr.

For further information see http://journals.iucr.org/services/authorrights.html

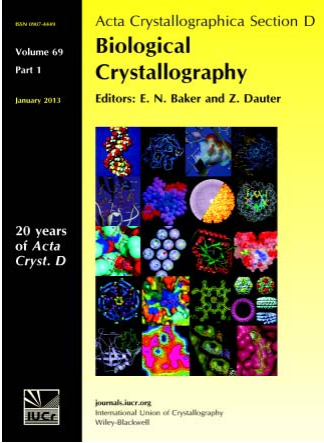

\begin{abstract}
Acta Crystallographica Section D: Biological Crystallography welcomes the submission of papers covering any aspect of structural biology, with a particular emphasis on the structures of biological macromolecules and the methods used to determine them. Reports on new protein structures are particularly encouraged, as are structure-function papers that could include crystallographic binding studies, or structural analysis of mutants or other modified forms of a known protein structure. The key criterion is that such papers should present new insights into biology, chemistry or structure. Papers on crystallographic methods should be oriented towards biological crystallography, and may include new approaches to any aspect of structure determination or analysis. Papers on the crystallization of biological molecules will be accepted providing that these focus on new methods or other features that are of general importance or applicability.
\end{abstract}

Crystallography Journals Online is available from journals.iucr.org 
Acta Crystallographica Section D

Biological

Crystallography

ISSN 0907-4449

John R. Helliwell* and Simon William Maurice Tanley

School of Chemistry, University of Manchester, Brunswick Street, Manchester M13 9PL, England

Correspondence e-mail:

john.helliwell@manchester.ac.uk

\section{The crystal structure analysis of the relative binding of cisplatin and carboplatin in a mixture with histidine in a protein studied at 100 and $300 \mathrm{~K}$ with repeated $X$-ray irradiation}

The anticancer agents cisplatin and carboplatin bind to histidine in a protein. This crystal structure study at datacollection temperatures of 100 and $300 \mathrm{~K}$ examines their relative binding affinities to a histidine side chain and the effect of a high X-ray radiation dose of up to $\sim 1.8 \mathrm{MGy}$ on the stability of the subsequent protein-Pt adducts. Cisplatin binding is visible at the histidine residue, but carboplatin binding is not. Five refined X-ray crystal structures are presented: one at $100 \mathrm{~K}$ as a reference and four at $300 \mathrm{~K}$. The diffraction resolutions are $1.8,2.0,2.8,2.9$ and $3.5 \AA$.

\section{Introduction}

Cisplatin and carboplatin (Supplementary Fig. S1 ${ }^{\mathbf{1}}$ ) are platinum anticancer drugs which bind to the N7 atoms of guanine bases in DNA, leading to inhibition of DNA replication and transcription and triggering apoptotic cell death (Benedetti et al., 2002; Silverman et al., 2002). However, 90\% of their reported binding is to plasma proteins (Fischer et al., 2008). Thus, these drugs cause serious side effects to patients. Cisplatin is rapidly converted to toxic metabolites which cause nephrotoxic effects (Zhang \& Lindup, 1996; Huličiak et al., 2012), whereas carboplatin has a slower rate of conversion to toxic metabolites owing to the addition of the cyclobutanedicarboxylate (CBDC) moiety (Supplementary Fig. S1) and hence is tolerated at higher chemical doses compared with cisplatin (Kostova, 2006). Even though these side effects are observed, both cisplatin and carboplatin remain in use for the treatment of testicular, bladder, ovarian and lung cancers, to name but a few (Wang \& Lippard, 2005; Ivanov et al., 1998). Cisplatin treatment is usually given to patients in combination with radiation therapy (Reedijk, 2003; Wang \& Lippard, 2005; Vallerga et al., 2004) as this drug is a radiation sensitizer (Peters et al., 2000), with standard treatment being to alternate between chemotherapy and radiation therapy. The concurrent treatment of cancers with both radiation therapy and cisplatin administration causes an above-additive effect, in which interaction with the radiation field leads to increased killing of cancer cells compared with each treatment individually (Seiwert et al., 2007). The combination of cisplatin and radiation therapy uses several mechanisms to enhance cell killing, including the enhanced formation of toxic platinum intermediates in the presence of radiation-induced free radicals, inhibition of DNA repair, a radiation-induced increase in cellular platinum uptake and cell-cycle arrest (Lawrence et al., 2003; Kvols, 2005).

\footnotetext{
${ }^{1}$ Supplementary material has been deposited in the IUCr electronic archive (Reference: YT5050). Services for accessing this material are described at the back of the journal.
}

Received 14 August 2012 Accepted 25 October 2012

PDB References: cisplatin/ carboplatin mixture binding to HEWL, 4gcb; 4gcc; 4gcd; 4gce; 4gcf 
Table 1

Crystallographic and refinement parameters of all data sets collected using the Bruker internal software programs.

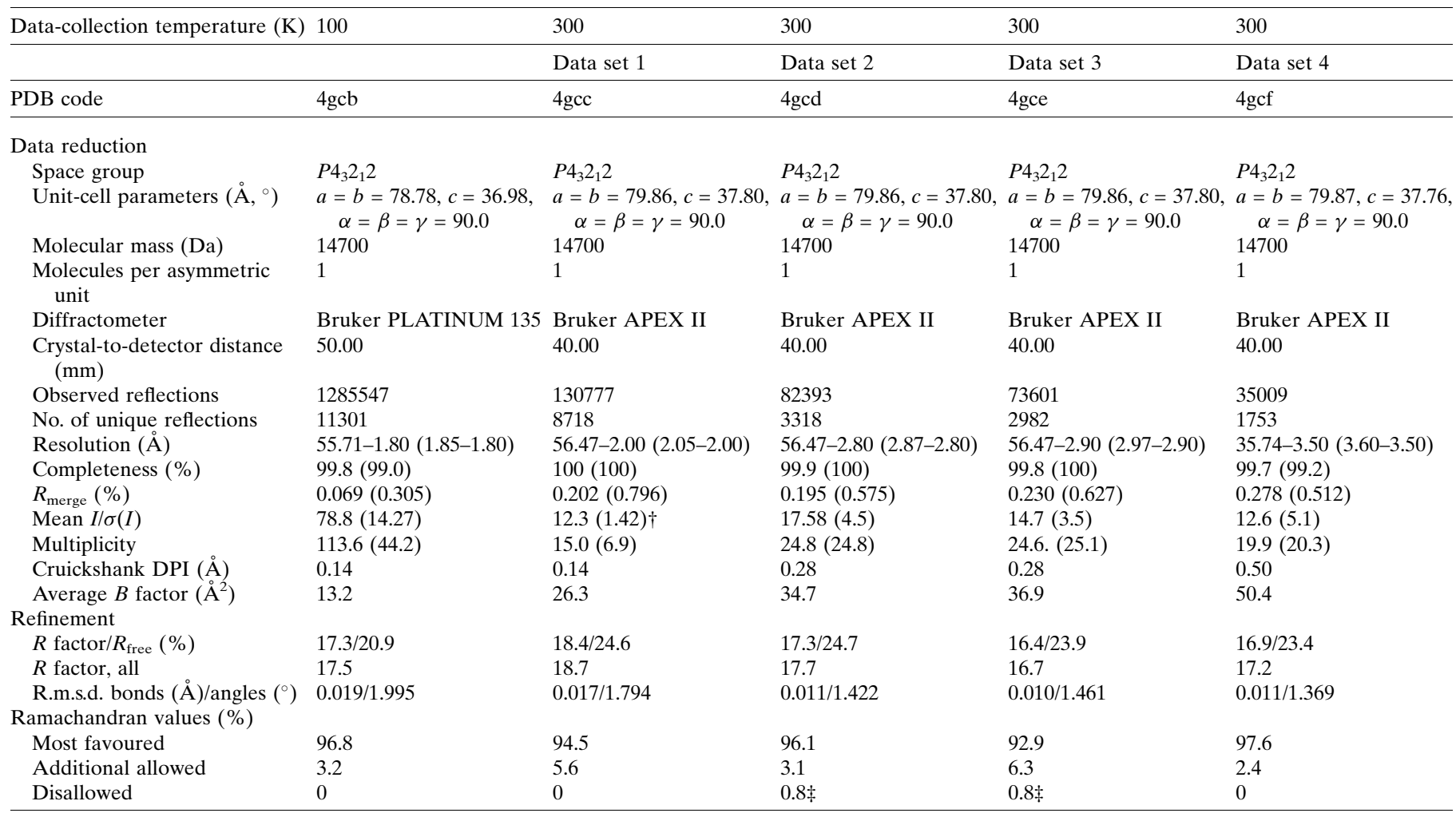

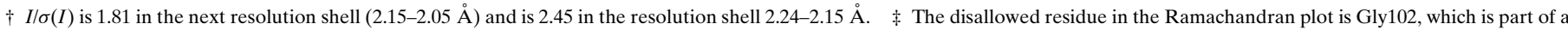
loop region of the protein.

In terms of crystallography, a previous study (Tanley, Schreurs, Kroon-Batenburg, Meredith et al., 2012) has shown that cisplatin and carboplatin bind to both the $\mathrm{N}^{\delta}$ and $\mathrm{N}^{\varepsilon}$ atoms of His15 in hen egg-white lysozyme (HEWL), a model protein, in DMSO medium after $8 \mathrm{~d}$ of cocrystallization. A further study (Tanley, Schreurs, Kroon-Batenburg \& Helliwell, 2012) used both $300 \mathrm{~K}$ X-ray diffraction data collection and $100 \mathrm{~K}$ X-ray diffraction data collection for carboplatin/ DMSO crystals after 13 months of chemical exposure. More detail was observed at the $\mathrm{N}^{\varepsilon}$ binding site for the CBDC moiety of carboplatin in DMSO conditions in the $300 \mathrm{~K}$ data set, whereas the cryo data sets after $8 \mathrm{~d}$ of cocrystallization (Tanley, Schreurs, Kroon-Batenburg, Meredith et al., 2012) and 13 months of chemical exposure (Tanley, Schreurs, KroonBatenburg \& Helliwell, 2012) were near-identical structurally. However, no structural differences were observed in the cisplatin binding sites on data collection at either 100 or $300 \mathrm{~K}$.

This further study focuses on the relative binding affinities of the Pt compounds cisplatin and carboplatin to a histidine side chain in a model protein (HEWL) in DMSO medium to determine which compound binds to the His 15 residue in a competitive situation and the effect of a high X-ray radiation dose on the stability of the subsequent protein-Pt adducts. Both 100 and $300 \mathrm{~K}$ X-ray diffraction data collections were used on separate crystals from the same crystallization pot to confirm whether more detail could be observed in one or the other crystal structure. Four X-ray diffraction (i.e repeated) data sets were collected from the same crystal at $300 \mathrm{~K}$ in order to understand the relative binding affinities of the mixture of cisplatin and carboplatin under continued X-ray irradiation. It is known that cisplatin and carboplatin are radiation sensitizers, as described above, and thus they are believed to be chemically stable during radiation therapy, which increases their activity in killing tumour cells by binding to DNA. The radiation reduction of bis(1-ethylimidazole)tartratoplatinum(II) complexes in methanol/water solutions has been examined (Kalecinka et al., 1997); this study found that the yields of platinum(I) depended on the presence of $\mathrm{O}_{2}$ in solution as well as the dose applied (up to $2 \mathrm{kGy}$ ). Our study reported here provides direct experimental structural details of the X-ray radiation-sensitive nature of cisplatin when bound to a model protein at $300 \mathrm{~K}$, which is important for directly determining the relative binding affinities of this anticancer drug to a model protein as well as the radiation stability of the histidine and its complexation.

\section{Methods}

\subsection{Crystallization conditions}

$49 \mathrm{mg}$ HEWL (3.2 mmol) was cocrystallized using the batch method with both $3 \mathrm{mg}$ cisplatin $(10 \mathrm{mmol})$ and $3.7 \mathrm{mg}$ carboplatin $(10 \mathrm{mmol})$ as well as $0.04 \mathrm{M}$ sodium acetate buffer $\mathrm{pH} 4.7$ (in $462.5 \mu \mathrm{l}$ ) and $10 \% \mathrm{NaCl}$ precipitant (in $462.5 \mu \mathrm{l}$ ) 
with $1 \mathrm{~m} M$ DMSO $(75 \mu \mathrm{l})$ and left at $277 \mathrm{~K}$. An overall sixfold molar concentration of the $\mathrm{Pt}$ compounds over that of the protein was used.

\subsection{X-ray data collection, structure solution and refinement}

For the cryo data collection, the Bruker temperaturecontrol device was set to $100 \mathrm{~K}$ and a crystal of $0.15 \mathrm{~mm}$ in size after $8 \mathrm{~d}$ of crystal growth was cryoprotected using silicone oil and centred on an in-house Bruker PLATINUM 135 detector at a distance of $50 \mathrm{~mm}$ from the detector. An X-ray exposure time of $10 \mathrm{~s}$ per $0.5^{\circ}$ crystal rotation range was used with an $\mathrm{X}$-ray wavelength of $1.5418 \AA$.

For the room-temperature (RT) data collection, the Bruker temperature-control device was set to $300 \mathrm{~K}$ and a crystal of $0.15 \mathrm{~mm}$ in size after $10 \mathrm{~d}$ of crystal growth was mounted in a $1 \mathrm{~mm}$ quartz capillary tube and centred on an in-house Bruker APEX II detector at a distance of $40 \mathrm{~mm}$ from the detector. Each data set consisted of a full $360^{\circ}$ rotation of the crystal comprising $40 \mathrm{~s} \mathrm{X}$-ray exposure per $0.5^{\circ}$ crystal rotation range for data sets 1 and 2 and $60 \mathrm{~s}$ per $0.5^{\circ}$ crystal rotation range for data sets 3 and 4 with an X-ray wavelength of $1.5418 \AA . \varphi$ scans were used for data collection in all cases (Tanley, Schreurs, Kroon-Batenburg \& Helliwell, 2012).

The $100 \mathrm{~K}$ data set was processed using the PROTEUM2 internal software package on the Bruker PLATINUM 135 detector. The $300 \mathrm{~K}$ data sets were processed using the $A P E X$ II internal software package on the Bruker APEX II detector; all four data sets from the $300 \mathrm{~K}$ data collection were processed and integrated separately. The unit-cell parameters were checked for X-ray damage effects, which showed possible significant changes for RT data set 4 (see Supplementary Table S1). All crystal structures were solved using molecular replacement with Phaser (McCoy et al., 2007) and restrained refinement with TLS (except for the $100 \mathrm{~K}$ data set, which was solved with restrained refinement only and no TLS) in REFMAC5 (Vagin \& Teplyakov, 2010) in CCP4 using the

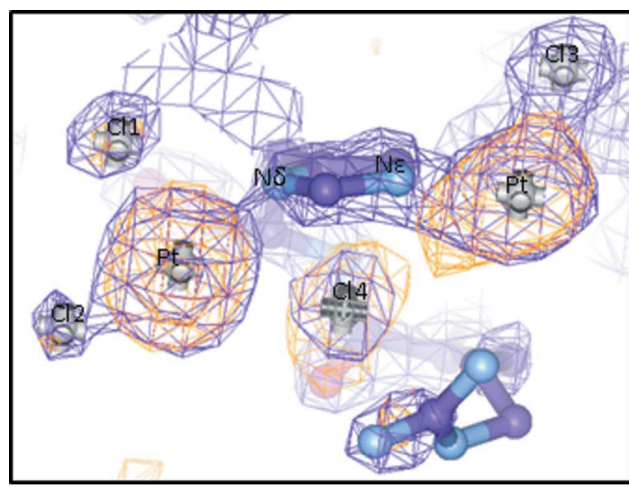

(a)

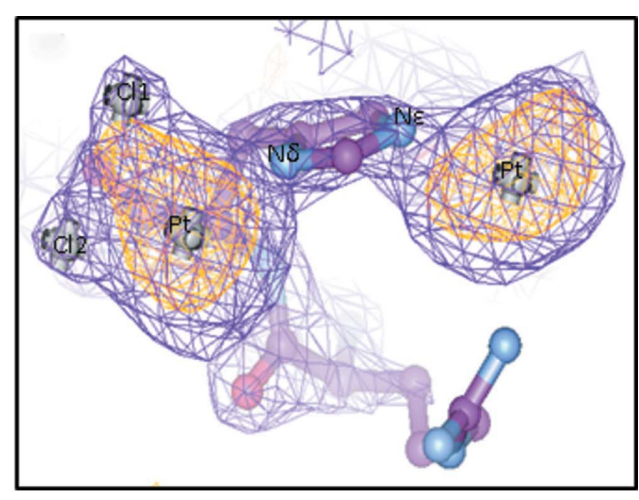

(b)
Figure 1

Cisplatin binding to the $\mathrm{N}^{\delta}$ and $\mathrm{N}^{\varepsilon}$ atoms of His15. (a) $100 \mathrm{~K}$ data collection at $1.8 \AA$ A resolution, (b) $300 \mathrm{~K}$ data set 1 at $2.0 \AA$ resolution. The $2 F_{\mathrm{o}}-F_{\mathrm{c}}$ map (in purple) is at the 1.5 r.m.s. cutoff level and the anomalous difference density map (in orange) is at the $3 \sigma$ cutoff level. The $\mathrm{N}^{\delta}$ and $\mathrm{N}^{\varepsilon}$ atoms in the imidazole ring are labelled, together with the $\mathrm{Pt}$ and $\mathrm{Cl}$ atoms of the bound cisplatin moiety. The $\mathrm{Cl}$ occupancies calculated from SHELXTL are 73 and $51 \%$ for $\mathrm{Cl} 1$ and $\mathrm{Cl} 2$ in the $100 \mathrm{~K}$ data set and 78 and $68 \%$ for $\mathrm{Cl} 1$ and $\mathrm{Cl} 2$ in the $\mathrm{RT}$ data set $1 . \mathrm{Cl} 3$ and $\mathrm{Cl} 4$ have occupancies of 25 and $36 \%$, respectively, in the $100 \mathrm{~K}$ data set.
Table 2

The Pt-occupancy values (\%) calculated from SHELXTL (Sheldrick, 2008).

Based on Tanley, Schreurs, Kroon-Batenburg, Meredith et al. (2012), the sigmas on these occupancies are estimated to be $\sim 5 \%$.

\begin{tabular}{lll}
\hline & $\mathrm{N}^{\delta}$ binding site & $\mathrm{N}^{\varepsilon}$ binding site \\
\hline 100 K data collection & 53 & 40 \\
300 K data collection & & \\
Data set 1 & 65 & 64 \\
Data set 2 & 64 & 64 \\
Data set 3 & 67 & 56 \\
Data set 4 & 71 & 59 \\
\hline
\end{tabular}

reported lysozyme structure 2w1y (Cianci et al., 2008) as a molecular search model. Model building, adjustment and refinement were carried out using the Coot (Emsley \& Cowtan, 2004) molecular-graphics program and REFMAC5 (Vagin \& Teplyakov, 2010), respectively, in CCP4. Crystallographic and refinement parameters are summarized in Table 1. Ligand-binding occupancies were calculated using SHELXTL (Sheldrick, 2008) and are given in Table 2.

\section{Results}

\subsection{Binding at His15}

Cisplatin rather than carboplatin is observed bound to both the $\mathrm{N}^{\delta}$ and $\mathrm{N}^{\varepsilon}$ atoms of the imidazole ring of His 15 in both the 100 and $300 \mathrm{~K}$ data sets. Fig. 1 shows the details of this binding of cisplatin at $100 \mathrm{~K}$ (Fig. 1a) and for the first data set collected at $300 \mathrm{~K}$ (Fig. $1 b$ ). In the $\mathrm{N}^{\delta}$ binding site, clear anomalous difference electron density is observed for the $\mathrm{Cl}$ atoms in the $100 \mathrm{~K}$ structure, with each atom being distinct, whereas for the $300 \mathrm{~K}$ data set 1 the $\mathrm{Cl}$ atoms are less well defined, but some anomalous electron density is also observed for them here. However, the $\mathrm{N}^{\varepsilon}$ binding site is more difficult to assign as either cisplatin or carboplatin. In the $100 \mathrm{~K}$ data set a distinct $\mathrm{Cl}$ atom is observed, but the anomalous difference density for this atom is weak. Data set 1 collected at $300 \mathrm{~K}$ shows less detail in the $\mathrm{N}^{\varepsilon}$ binding site; hence, only a $\mathrm{Pt}$ ion can be assigned into the density. The other three data sets collected at $300 \mathrm{~K}$ were collected to lower resolutions (2.8, 2.9 and $3.5 \AA$ ); they also all show clear binding to both binding sites (Supplementary Fig. S2) supported by anomalous difference electron density for the Pt ion, but the data are of poorer quality owing to overall radiation damage. Hence, the data are too poor to provide detailed information as to the coordinated atoms beyond the $\mathrm{Pt}$ ion. Hence, it is difficult to assign whether cisplatin or carboplatin is 
present, but owing to the observation of cisplatin binding in the $100 \mathrm{~K}$ structure it is assumed that cisplatin rather than carboplatin is also bound in the $300 \mathrm{~K}$ structure. A whole cisplatin molecule was initially centred on the $\mathrm{Pt}$ position for both the $\mathrm{N}^{\delta}$ and $\mathrm{N}^{\varepsilon}$ binding sites and its coordinated atoms were removed based on electron density not being observed. The $\mathrm{Pt}-\mathrm{His} \mathrm{N}$ distances were not restrained during refinement. The occupancy values of the Pt ions at each binding site in the $100 \mathrm{~K}$ and the four $300 \mathrm{~K}$ data sets are given in Table 2.

The data set at $100 \mathrm{~K}$ has an extra atom visible near the Arg14 residue (Fig. 1a). This is assumed to be likely to be a $\mathrm{Cl}$ atom. HEWL has the six usual chloride-binding sites and in this $100 \mathrm{~K}$ data set these have anomalous difference electrondensity peak heights of between $5 \sigma$ and $11 \sigma$, with the new site having a peak-height value of $9.6 \sigma$. However, the $2 F_{\mathrm{o}}-F_{\mathrm{c}}$ electron density for this Arg14 residue is weak and thus it is difficult to assign this density to the Arg14 side chain or to a molecule of water. Assuming the presence of an arginine, the $B$ factors are between 24 and $34 \AA^{2}$.

\subsection{Absorbed $X$-ray radiation dose for the $300 \mathrm{~K}$ crystal study}

The X-ray diffraction data collected at $300 \mathrm{~K}$ were partitioned into four data sets, as described in \$2.2. This datacollection strategy was used to confirm how stable the cisplatin/carboplatin molecule is when bound to a histidine residue in a model protein, as it is known that these drugs are radiation sensitizers and thus are used in conjunction with radiation therapy. This crystal was not 'back-washed' before data collection; hence, the solvent channels of the protein crystal contained $\mathrm{Pt}$ ions. Taking into account explicitly the solvent content as well as the protein content, and the bound cisplatin and DMSO molecules to the protein, the absorbed $\mathrm{X}$-ray radiation dose is $0.36 \mathrm{MGy}$ for data sets 1 and 2 and $0.53 \mathrm{MGy}$ for data sets 3 and 4 . This thus gives an overall absorbed radiation dose of $1.78 \mathrm{MGy}$ for this crystal. The dose calculation and equations used are given in Supplementary Table S3. Supplementary Fig. S3 shows the $F_{\mathrm{o}}-F_{\mathrm{c}}$ electrondensity maps after rigid-body refinement for all four data-set runs but with data sets 1,2 and 3 cut at $3.5 \AA$ resolution (the resolution limit of data set 4 ) and Supplementary Fig. S4 shows data sets 1 and 2 cut at $2.9 \AA$ resolution (the resolution limit of data set 3 ). These figures confirmed that the electrondensity detail for each data-set experiment is exactly the same, which was confirmed by the Pt-occupancy values (Table 2). The occupancies do differ slightly, but they are not greatly different and are in the range $\pm 5 \%$ (Tanley, Schreurs, KroonBatenburg, Meredith et al., 2012); this is thus in agreement with the principle that cisplatin is a radiation sensitiser and is stable during repeated X-ray irradiation. Furthermore, we observed that its binding to histidine remained throughout this $\mathrm{X}$-ray dose.

\section{Discussion}

This study uses the same molar concentration of cisplatin and carboplatin to determine which of the anticancer compounds has higher affinity for binding to a histidine residue in a model protein. Additionally, this study also looked at the stability of the bound compound (cisplatin) to continued X-ray irradiation. Finally, the stability of the attachment of cisplatin to His15 is assessed. At the diffraction resolutions used here (i.e $\sim 2 \AA$ ), it is difficult to detect whether any reduction of $\mathrm{Pt}^{2+}$ to $\mathrm{Pt}^{+}$occurs in this study; this would have to be assessed in a future study at a higher resolution.

\subsection{Cisplatin rather than carboplatin binding is preferred}

A threefold molar excess of both cisplatin and carboplatin was used in the cocrystallization with HEWL, giving an overall sixfold molar excess of the $\mathrm{Pt}$ compounds over the protein. Both the 300 and $100 \mathrm{~K}$ X-ray diffraction data sets showed evidence for binding of cisplatin over carboplatin in the $\mathrm{N}^{\delta}$ binding site, as indicated by the presence of anomalous difference electron density for the $\mathrm{Cl}$ atoms bound to the $\mathrm{Pt}$ ion (Figs. $1 a$ and $1 b$ ). Cisplatin also bound to the $\mathrm{N}^{\varepsilon}$ binding site in the $100 \mathrm{~K}$ structure (Fig. 1a), as again indicated by the presence of anomalous difference electron density for a $\mathrm{Cl}$ atom. However, for the $300 \mathrm{~K} \mathrm{X}$-ray diffraction data sets it was difficult to assign which $\mathrm{Pt}$ compound bound to the $\mathrm{N}^{\varepsilon}$ atom owing to the deteriorating quality of the data under repeated $\mathrm{X}$-ray irradiation, meaning that only a $\mathrm{Pt}$ ion could be modelled into the density and no specific coordinated species could be assigned. However, owing to the fact that cisplatin rather than carboplatin was observed to be clearly bound in the $100 \mathrm{~K}$ structure, it can be deduced that cisplatin does have an overall higher binding affinity for the His side-chain $\mathrm{N}^{\delta}$ and $\mathrm{N}^{\varepsilon}$ atoms compared with carboplatin in this model study. For the binding of two Pt ions to occur to the imidazole ring of this histidine side chain, both of the $\mathrm{N}$ atoms are $s p^{2}$-hybridized with $\mathrm{N}$ lone pairs in the plane of the imidazole ring, providing two $\mathrm{N}$ atoms to which a metal centre can bind (Tanley, Schreurs, Kroon-Batenburg, Meredith et al., 2012). The usual $\mathrm{N}$-hydrogen of one of the histidine $\mathrm{N}$ atoms has to be removed and this could be facilitated by the crystallization conditions used, which contained both chloride and acetate ions.

\subsection{Absorbed $X$-ray radiation dose of the $300 \mathrm{~K}$ crystal}

This study also looked at the stability of cisplatin towards continued X-ray irradiation once bound to the histidine side chain. Under continued X-ray irradiation in the $300 \mathrm{~K}$ study, the crystal absorbed 1.78 MGy of radiation (Supplementary Table S3) over the four X-ray diffraction data sets collected, and the electron-density maps for the bound cisplatin molecules looked very similar even at the differing resolutions of each data set (Supplementary Figs. S3 and S4). The results confirmed that cisplatin is stable over prolonged exposure to $\mathrm{X}$-ray irradiation as it does not dissociate from the histidine residue, meaning that its relative binding affinity to this model protein is fixed. This result was backed up by the Pt-occupancy values for each $300 \mathrm{~K} \mathrm{X}$-ray diffraction data set (Table 2), which do not vary significantly (they are within $\pm 5 \%$ ) between each run. Hence, the relative binding affinity of cisplatin to 
HEWL, our model protein, has not been affected by the quantity of X-ray radiation absorbed.

\section{Conclusions}

This study showed that cisplatin has a higher relative binding affinity than carboplatin for binding to a histidine side chain in a model protein. Also, cisplatin was stable as a chemical structure upon continued X-ray irradiation; continued binding of cisplatin to the histidine side chain was observed even after prolonged irradiation. This means that the relative binding affinity of cisplatin to a protein is the same at the end of the range of X-ray absorbed dose used in this study. Obviously, it should be mentioned that the chemical mixtures used in the crystallization chemical conditions required for crystallography are not the same as those used when treating a cancer patient; likewise, the precise photon-energy and X-ray dose used in this study are different (see Supplementary Table S3). These differences impose a limitation on the relevance of the conclusions reached here in terms of a cancer treatment scenario.

JRH is grateful to the University of Manchester for general support, to the ESPRC for a PhD studentship to SWMT and to the School of Chemistry for crystallization, computing and $\mathrm{X}$-ray facilities. We are grateful to Dr Chris Muryn of the School of Chemistry for his stewardship of the X-ray CCD APEX II diffractometer.

\section{References}

Benedetti, M., Malina, J., Kasparkova, J., Brabec, V. \& Natile, G. (2002). Environ. Health Perspect. 110, Suppl. 5, 779-782.
Cianci, M., Helliwell, J. R. \& Suzuki, A. (2008). Acta Cryst. D64, 1196-1209.

Emsley, P. \& Cowtan, K. (2004). Acta Cryst. D60, 2126-2132.

Fischer, S. J., Benson, L. M., Fauq, A., Naylor, S. \& Windebank, A. J. (2008). Neurotoxicology, 29, 444-452.

Huličiak, M., Vacek, J., Sebela, M., Orolinová, E., Znaleziona, J., Havlíková, M. \& Kubala, M. (2012). Biochem. Pharmacol. 83, 1507-1513.

Ivanov, A. I., Christodoulou, J., Parkinson, J. A., Barnham, K. J., Tucker, A., Woodrow, J. \& Sadler, P. J. (1998). J. Biol. Chem. 273, 14721-14730.

Kalecinka, E., Kalecinski, I. J. \& Kuduk-Jaworsk, J. (1997). Radiat. Phys. Chem. 50, 381-384.

Kostova, I. (2006). Recent Pat. Anticancer Drug. Discov. 1, 1-22.

Kvols, L. K. (2005). J. Nucl. Med. 46, 187S-190S.

Lawrence, T. S., Blackstock, A. W. \& McGinn, C. (2003). Semin. Radiat. Oncol. 13, 13-21.

McCoy, A. J., Grosse-Kunstleve, R. W., Adams, P. D., Winn, M. D., Storoni, L. C. \& Read, R. J. (2007). J. Appl. Cryst. 40, 658-674.

Peters, W. A. III, Liu, P. Y., Barrett, R. J. II, Stock, R. J., Monk, B. J., Berek, J. S., Souhami, L., Grigsby, P., Gordon, W. Jr \& Alberts, D. S. (2000). J. Clin. Oncol. 18, 1606-1613.

Reedijk, J. (2003). Proc. Natl Acad. Sci. USA, 100, 3611-3616.

Seiwert, T. Y., Salama, J. K. \& Vokes, E. E. (2007). Nature Clin. Pract. Oncol. 4, 86-100.

Sheldrick, G. M. (2008). Acta Cryst. A64, 112-122.

Silverman, A. P., Bu, W., Cohen, S. M. \& Lippard, S. J. (2002). J. Biol. Chem. 277, 49743-49749.

Tanley, S. W. M., Schreurs, A. M. M., Kroon-Batenburg, L. M. J. \& Helliwell, J. R. (2012). Acta Cryst. F68, 1300-1306.

Tanley, S. W. M., Schreurs, A. M. M., Kroon-Batenburg, L. M. J., Meredith, J., Prendergast, R., Walsh, D., Bryant, P., Levy, C. \& Helliwell, J. R. (2012). Acta Cryst. D68, 601-612.

Vagin, A. \& Teplyakov, A. (2010). Acta Cryst. D66, 22-25.

Vallerga, A. K., Zarling, D. A. \& Kinsella, T. J. (2004). Clin. Adv. Hematol. Oncol. 2, 793-805.

Wang, D. \& Lippard, S. J. (2005). Nature Rev. Drug Discov. 4, 307-320.

Zhang, J.-G. \& Lindup, W. E. (1996). Toxicol. Lett. 89, 11-17. 